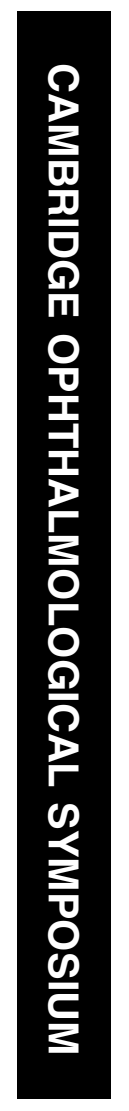

\section{Angiogenesis and tumour progression: migration- stimulating factor as a novel target for clinical intervention}

\begin{abstract}
Migration-stimulating factor (MSF), a soluble genetically truncated isoform of fibronectin, is a potent oncofoetal regulatory molecule. Its 2.1-kb message is generated from the fibronectin gene by a variant of standard alternative splicing involving premature intraintronic cleavage. MSF is constitutively expressed by both epithelial and stromal cells during foetal development and in patients with cancer, but is generally not expressed in healthy adults. MSF affects the behaviour of a broad range of potential target cells (fibroblasts, vascular, and epithelial) in terms of stimulation of their migration/invasion, matrix remodelling and induction of
\end{abstract}

Unit of Cell and Molecular Biology, The Dental School, University of Dundee, Dundee, Scotland, UK

Correspondence: AM Schor, Unit of Cell and Molecular Biology, The Dental School, University of Dundee, Dundee DD1 4HR, UK Tel: + 01382 635987; Fax: + 01382635998 E-mail: a.m.schor@ dundee.ac.uk

Received: 29 September 2009

Accepted: 17 November 2009

Published online:

18 December 2009

Review presented at the 39th Cambridge Ophthalmological Symposium. Cambridge, 2-4 September 2009 to be published in Eye, March 2010
AM Schor and SL Schor

means of improving treatment outcome in cancer patients.

Eye (2010) 24, 450-458; doi:10.1038/eye.2009.314; published online 18 December 2009

Keywords: cancer; metastasis; MSF; angiogenesis; epigenetics; 5-azacytidine

Tumour-induced angiogenesis

The progressive enlargement and metastatic dissemination of an incipient tumour mass has long been recognised to be critically dependent on it acquiring the capacity to induce formation of new blood vessels from the extant tissue microvasculature, a process defined as 'tumourinduced angiogenesis'. ${ }^{1-3}$ The induction of an analogous angiogenic response is also fundamental to the aetiology of other general and ophthalmic pathologies, including fibrosis, rheumatoid arthritis, proliferative diabetic retinopathy, and age-related macular degeneration. ${ }^{4-6}$ The important contribution of vascular endothelial growth factor (VEGF) to the initiation of angiogenesis in these conditions is now well established, with beneficial outcomes of VEGF-targeted therapy beginning to be achieved in certain conditions. ${ }^{7,8}$

In spite of these encouraging results, it remains apparent that further basic and translational research is required for antiangiogenic therapy to realise its full clinical potential for patients with cancer ${ }^{9-11}$ and for neovascular-driven ophthalmic pathologies. ${ }^{12,13}$ This ongoing need stems from the fact the initiation, directionality, and maintenance of angiogenesis is dictated by the dynamic balance between a multitude of pro- and antiangiogenic stimuli. ${ }^{1,2,14,15}$ In view of the 
inherently complex and potentially redundant nature of this tissue-level (contextual) control network, it is unlikely that VEGF, or indeed any single stimulus, will afford a generally efficacious anti-angiogenic target.

We have identified and cloned migration-stimulating factor (MSF), a novel motogenic/angiogenic factor, which seems to have a significant role in driving tumour progression. Our initial results further indicate that the manifestation of MSF bioactivity (in terms of both target cell selection and specific response) is stringently modulated by a network of contextual controls, including the presence of other soluble factors and the physico-chemical nature of the extracellular matrix (ECM). In this brief review, we discuss MSF in terms of (1) its molecular characterisation and diverse functionality, (2) the influence of contextual parameters in defining both its expression and effects on potential target cells, and (3) its possible use as a novel target for clinical intervention.

\section{MSF: molecular characterisation and diverse functionality}

MSF is an oncofoetal protein initially shown to be constitutively expressed in vitro by foetal and cancer patient fibroblasts, but not by their healthy adult counterparts. ${ }^{16,17}$ Unexpectedly, MSF-expressing fibroblasts were obtained from both tumour-associated ${ }^{18}$ and distant uninvolved sites in cancer patients, thereby indicating the systemic nature of this abnormality. ${ }^{19}$ Subsequent ex vivo studies have indicated that both MSF message and protein are expressed by fibroblasts, keratinocytes, and vascular endothelial cells in foetal skin, but not by the majority of these cells in the healthy adult skin. ${ }^{20}$ MSF is expressed by tumour and tumourassociated stromal cells in the majority of tumours examined to date, including common carcinomas, melanomas, and gliomas ${ }^{20,21}$ (unpublished observations).

MSF is the first genetically truncated isoform of human fibronectin to be identified. ${ }^{20}$ Approximately 20 'full-length' fibronectin isoforms had already been characterised when MSF was cloned. All of these consist of two peptide chains (250-280 kDa) covalently linked by disulphide bonds at their respective $C$ termini. ${ }^{22}$ The expression of MSF is controlled at the posttranscriptional level by a two-step mechanism. The primary MSF transcript is initially generated from the single-copy fibronectin gene by read through of the intron separating exons III- $1 \mathrm{a}$ and III- $1 \mathrm{~b}$, followed by intra-intronic cleavage to produce a 5.9-kb MSF pre-message. ${ }^{23}$ The pre-message remains sequestered within the nucleus, where it is rapidly degraded as a consequence of an AU-rich instability element in its $3^{\prime}$-UTR. ${ }^{20,23,24}$ In cells expressing the MSF protein, the intron-derived $3^{\prime}$-sequence of the pre-message is cleaved for a second time to produce a $2.1-\mathrm{kb}$ mature MSF message. This has a significantly shorter (195 bp) intron-derived $3^{\prime}$-sequence containing a 30-bp in-frame coding sequence (immediately contiguous with exon III-1a), followed by a 165-bp 3'-UTR containing several in-frame stop codons and a polyadenylation signal. The mature message is rapidly exported to the cytoplasm for translation. MSF is consequently a $70-\mathrm{kDa}$ protein identical to the $\mathrm{N}$ terminus of a full-length fibronectin monomer (up to and including the amino-acid sequence coded by exon III-1a), with the addition of an MSF-unique (intron-coded) 10 amino-acid C terminus (Figure 1).

Zhao et $a l^{25}$ described a similarly foreshortened fibronectin message in Zebrafish embryos. Truncated fibronectin isoforms have also been detected in cDNA libraries from goldfish and rainbow trout, as well as from mouse and human liver, prostate, ovary, brain, and spleen. ${ }^{26}$ Using a polyclonal anti-murine MSF, we have recently documented the expression of MSF protein in mouse tissues during foetal development (C. Carr, P. Felts et al, unpublished observations).

MSF is a potent motogen, stimulating the migration of epithelial cells (normal and tumour), as well as adultderived skin fibroblasts, vascular endothelial cells, and pericytes (Figure 2). These cells show a bell-shaped dose response with half-maximal activity manifesting at $0.1-1.0 \mathrm{pg} / \mathrm{ml}$, that is, femtomolar concentrations. MSF also stimulates the migration of non-epithelial tumour cells and displays other bioactivities relevant to tumour progression, such as the upregulation of hyaluronan (HA) synthesis, proteolysis, and induction of angiogenesis ${ }^{20,21,27-29}$ (unpublished observations). The angiogenic activity of MSF has been demonstrated in both in vitro and in vivo models. For example, MSF induces endothelial activation in vitro, as manifested by the adoption of a sprouting cell phenotype (Figure 3). It also induces angiogenesis in rats (Figure 4) and pigs when implanted subcutaneously, and in the chick embryo assay (Figure 5). In the latter, different quantities of MSF were incorporated within dried methyl cellulose gels (MCGs), which were then applied to the yolk-sac membrane. The induction of a subjacent radial array of blood vessels $24 \mathrm{~h}$ later was considered a positive angiogenic response. MSF elicited a significant (bellshaped) angiogenic response over a broad concentration range of $0.5-500 \mathrm{ng} / \mathrm{MCG}$.

The IGD tripeptide motif (isoleucine-glycineaspartate) is a highly conserved feature of fibronectin type I modules to which no biological functionality was initially ascribed. ${ }^{22}$ MSF contains four such IGD motifs (Figure 1). In vitro mutagenesis studies ${ }^{20}$ have indicated that the stimulation of fibroblast migration by MSF is mediated by the two IGD motifs present in type I 


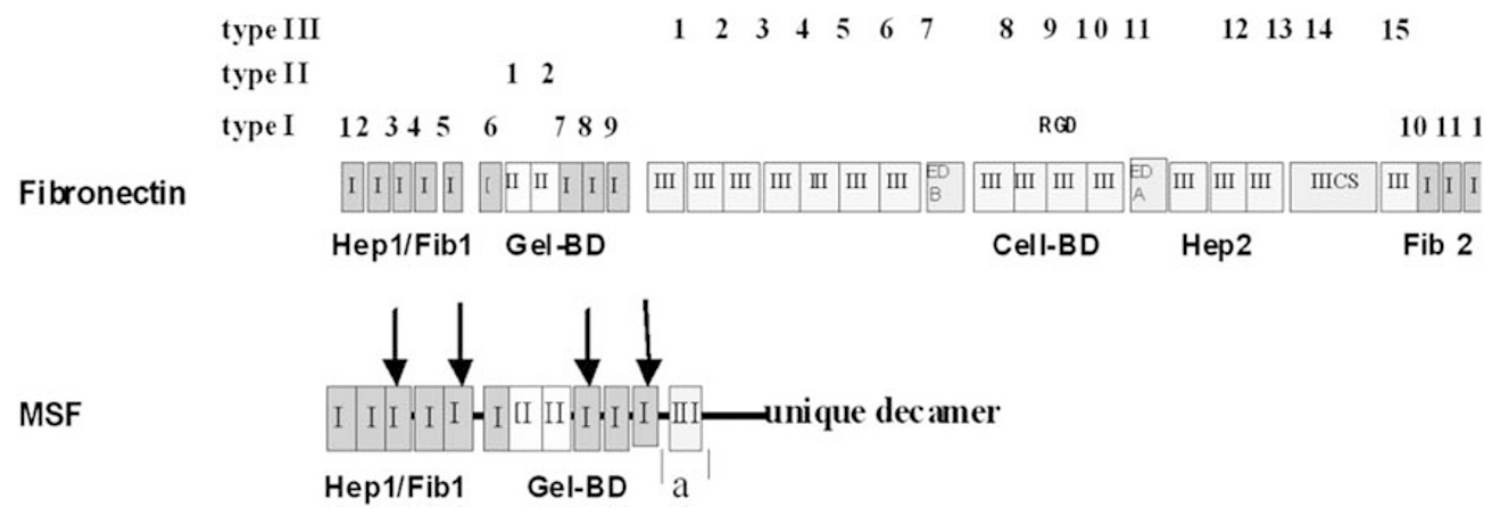

Figure 1 MSF is a genetically truncated isoform of fibronectin, which is a modular glycoprotein consisting of the following 'functional domains' defined on the basis of their characteristic binding affinities for other matrix macromolecules and cell-surface receptors: Hep-1/Fib-1 (N-terminal binding to heparin and fibrin), Gel-BD (binding to gelatin/collagen), Cell-BD (RGD-mediated binding to cell-surface integrins), Hep-2 (high-affinity binding to heparin), and Fib-2 (C-terminal binding to fibrin). Each of these functional domains is composed of several 'homology modules', designated as types I, II, and III, respectively. Alternative splicing at EDA, EDB, and IIICS generates 20 'full-length' fibronectin isoforms (molecular masses in the region of 250-280 kDa). MSF is a truncated $(70 \mathrm{kDa})$ isoform of fibronectin identical to its $\mathrm{N}$ terminus, up to and including the amino-acid sequence coded by exon III- $1 \mathrm{a}$ $\left(\right.$ Schor $e a^{20}$ ). MSF message is transcribed from the fibronectin gene by a variation of standard alternative splicing involving intron read through and premature transcript cleavage (Kay et $\mathrm{al}^{23}$ ). MSF consequently terminates in a unique 10 amino-acid (intron-derived) sequence not present in any full-length fibronectin. Arrows indicate the location of the four IGD motifs in modules I-3, I-5, I-7, and I-9.

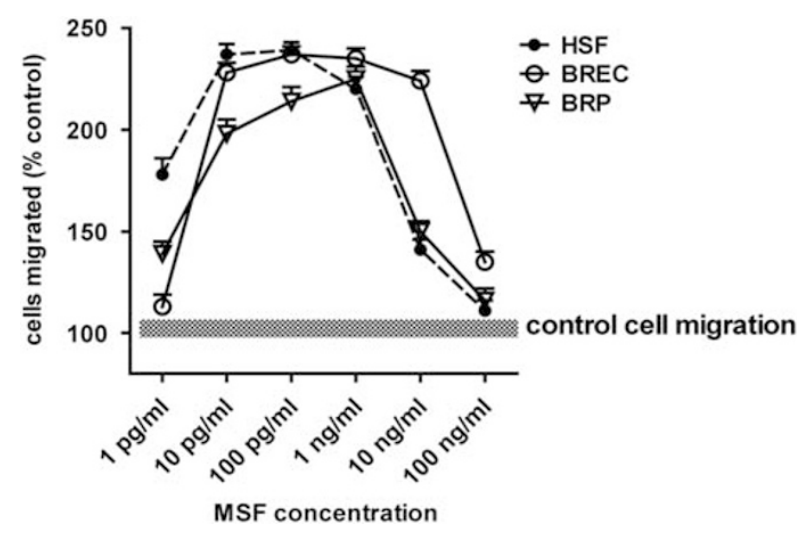

Figure 2 Dose-response stimulation of cell migration by MSF. The effects of different concentrations of MSF $(1 \mathrm{pg} / \mathrm{ml}-100 \mathrm{ng} / \mathrm{ml})$ on the migration of human skin fibroblasts (HSF), bovine retinal endothelial cells (BREC), and bovine retinal pericytes (BRP) were ascertained in the transmembrane (Boyden chamber) assay using membranes coated with native type I collagen. In these experiments, the number of migrated cells in the absence of MSF (control or baseline migration) was $20.7 \pm 1.1,15.5 \pm 0.6$, and $14.5 \pm 0.8$ (mean \pm SD) for BREC, BRP, and HSF, respectively. To compare different experiments, the number of migrated cells in the presence of MSF are expressed as a percentage of control values (horizontal shaded bar).

modules I-7 and I-9, whereas all four IGD motifs affect endothelial cell migration. Critically, small IGDcontaining synthetic peptides mimic all MSF bioactivities, including the stimulation of cell migration, endothelial cell activation, and angiogenesis (Figures $3-5)^{20,30}$ (unpublished observations). It is important to note that none of these bioactivities are manifested by any full-length fibronectin isoform, apparently as a consequence of steric hindrance. ${ }^{31,32}$ Houard et a ${ }^{29}$ have identified a second motif (HEEGH) in MSF module I-8 that seems to mediate the motogenic response of a breast cancer cell line (MCF7) and is additionally required for manifestation of its proteinase activity. Our recent data indicate that the motogenic response of certain target cells may be mediated by both IGD and HEEGH, whereas other cells only respond to the IGD motif.

The role of contextual tissue-level parameters in the control of MSF expression and manifestation of its bioactivities

The programmed control of gene expression during development has long been recognised to be regulated by epigenetic mechanisms. In contrast to their genetic (eg, mutational) counterparts, epigenetic mechanisms are mediated by reversible, although heritable (persistent), changes in DNA and/or histone methylation. We have recently demonstrated that MSF expression may be switched on and off in such a reversible and persistent manner. For example, a transient $(2 \mathrm{~h})$ exposure to TGF- $\beta 1$ of normal adult fibroblasts (non-producers of MSF protein) growing on a 'wounded' substratum in vitro (such as denatured type I collagen or fibrin) induces expression of the MSF protein. This 'activated' phenotype is persistent for the entire subsequent life span of the treated cells when cultured in the absence of TGF- $\beta$ and irrespective of the nature of the substratum. 


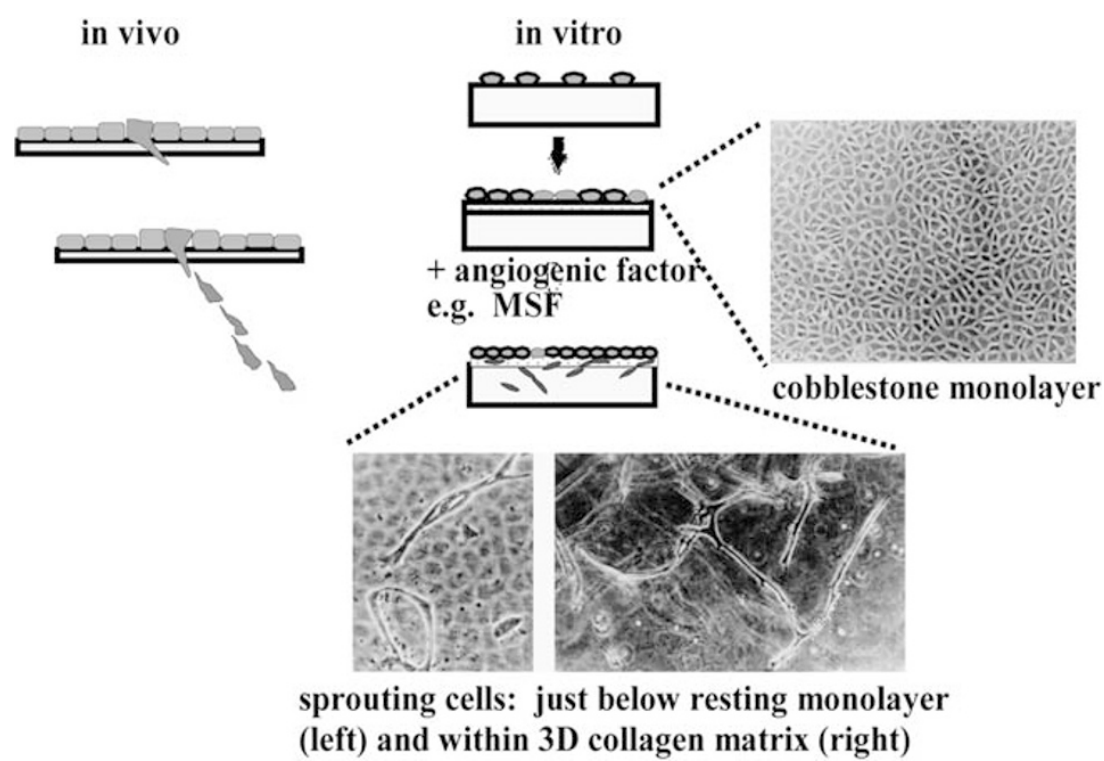

Figure 3 In vitro angiogenesis assay. Endothelial cells plated on the surface of a 3D collagen gel initially form a monolayer of 'resting' 'cobblestone' cells analogous to the cells lining the vessel lumen in vivo. This monolayer is maintained in culture in the absence of exogenous angiogenic factors. Addition of such a factor (eg, MSF or VEGF) results in the rapid induction of a network of cells displaying a 'sprouting' phenotype. These cells migrate down through the extracellular matrix deposited by the cobblestone cells and into the provided $3 \mathrm{D}$ collagen gel matrix.
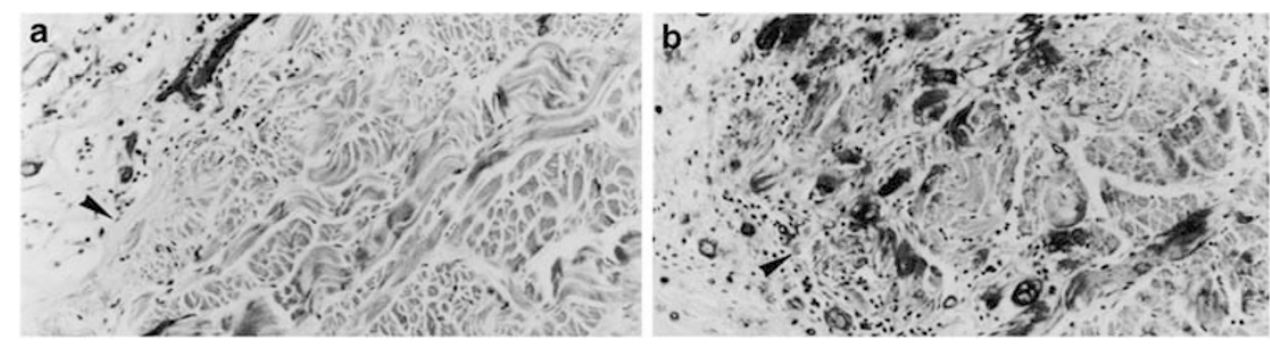

Figure 4 The induction of angiogenesis and fibroblast migration by MSF in the rat. Collagenous graft matrices were soaked for $24 \mathrm{~h}$ in either PBS buffer (control graft) or PBS containing $10 \mathrm{ng} / \mathrm{ml}$ MSF (MSF graft). These were then implanted subcutaneously in rats. Skin biopsies containing the grafts were excised, fixed, and paraffin-embedded 28 days after implantation. Arrowheads mark the edge of the grafts. (a) Control implant. Blood vessels (stained with biotinylated Griffonia simplicifolia lectin) and fibroblasts were principally confined to the host tissue surrounding the implant. (b) MSF implant. Significantly more blood vessels and fibroblasts have infiltrated the graft matrix in response to MSF.

However, MSF expression may be switched off again at any time by a second transient exposure to TGF- $\beta 1$, this time when the cells are growing on a 'healthy' matrix of native type I collagen (Figure 6). It is important to note that this 'on-off' switch is repeatable and strictly requires the concerted action of TGF- $\beta 1$ and the appropriate matrix; that is, exposure to TGF- $\beta 1$ of previously activated cells growing on a wounded matrix will not switch off MSF expression. Initial data consistent with the involvement of epigenetic mechanisms have been provided by observations that a transient exposure of normal adult fibroblasts to 5-azacytidine, a pharmacological agent inducing changes in gene expression by CpG island demethylation, results in a persistent switch on of the MSF expression; again, this induced expression may be switched off by a subsequent exposure of activated cells to TGF- $\beta 1$ when grown on a native collagen substratum (Figure 6). These matrixdependent effects of TGF- $\beta$ on MSF expression are consistent with numerous previous observations, indicating that the precise effects of TGF- $\beta$ on target cells is determined by the nature of the ECM and other contextual cues. ${ }^{33-36}$

The potential clinical relevance of these findings is highlighted by observations that constitutive MSF expression by foetal and cancer patient fibroblasts may 


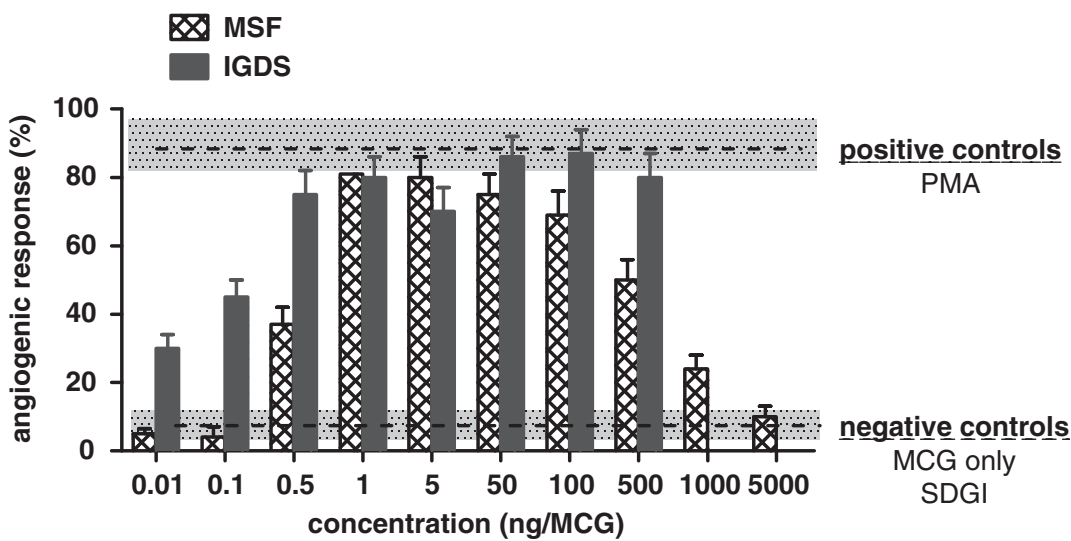

Figure 5 Induction of angiogenesis in the chick yolk-sac assay by MSF and IGDS. Different concentrations of MSF and IGDS were incorporated into methyl cellulose gels (MCGs) and these were then dried and applied to the chick embryo yolk-sac membrane. The induction of a radial disposition of vessels $24 \mathrm{~h}$ later was scored as a positive angiogenic response. Results are expressed as the percentage of positive angiogenic responses elicited by various concentrations of MSF and IGDS. A significant response was induced over a broad concentration range of $0.5-500 \mathrm{ng}$ per MCG for both agents. PMA ( $300 \mathrm{ng}$ per MCG) was used as positive control. Upper and lower horizontal bars indicate values achieved by the positive and negative controls, respectively (mean and SD).

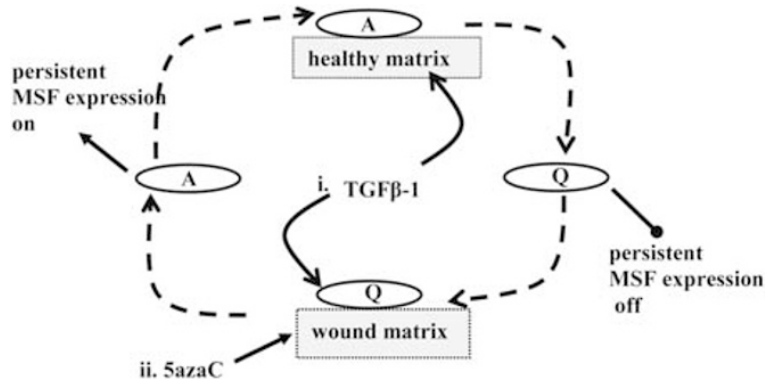

Figure 6 Control of MSF expression. Quiescent (Q) adult skin fibroblasts do not produce MSF. However, they are induced to do so by (i) a single transient ( $2 \mathrm{~h}$ ) exposure to TGF- $\beta 1$ when cultured on a 'wound' matrix, such as fibrin or denatured type I collagen. This switch on is persistent and the resultant 'activated' (A) cells continue to express MSF for the entire duration of their in vitro life span when cultured under standard tissue culture conditions in the absence of TGF- $\beta$ and irrespective of the nature of their substratum. MSF expression may be persistently switched off again by a subsequent transient exposure of activated cells to TGF- $\beta$, this time when they are grown on a 'healthy-tissue' matrix (such as native type I collagen). This switch on and off is completely reversible and may be repeated for the entire duration of their in vitro life span. Quiescent cells may be similarly activated by a transient exposure to (ii) 5-azacytidine (5azaC). The persistent MSF expression so induced is again switched off by a subsequent transient exposure of A cells to TGF- $\beta 1$ when adherent to a native collagen substratum.

also be persistently switched off by the exposure of cells growing on a native collagen substratum to TGF- $\beta 1$. The ability of potentially deleterious MSF expression to be switched off by pharmacological intervention suggests that it may be possible to develop novel

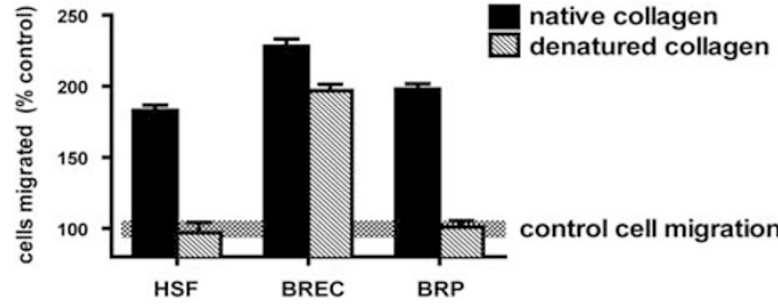

Figure 7 Matrix modulation of the motogenic response of target cells to MSF. The effects of the substratum (native or denatured type I collagen) on the motogenic response of human skin fibroblasts (HSF), bovine retinal endothelial cells (BREC), and bovine retinal pericytes (BRP) to $10 \mathrm{pg} / \mathrm{ml}$ MSF were evaluated in the transmembrane (Boyden chamber) migration assay. The number of cells migrated in the presence of MSF is expressed as a percentage of baseline (control) migration. BRP and HSF only responded to MSF when attached to a native collagen substratum. In contrast, the migration of BREC was stimulated by MSF irrespective of substratum.

therapeutic strategies on the basis of the modulation of MSF expression in a clinically desirable manner.

The functional response of target cells to MSF (and its IGD synthetic peptide mimetic) is also modulated by contextual parameters. For example, the stimulation of fibroblast migration is manifested by cells adherent to a native, but not denatured, type I collagen substratum. ${ }^{20,30}$ Native collagen may accordingly be considered to provide a 'permissive' substratum, whereas denatured collagen is 'non-permissive'. The motogenic response of pericytes, but not that of endothelial cells, exhibits similar matrix dependence (Figure 7). The stimulation of fibroblast migration by MSF is also abrogated by various soluble factors, 

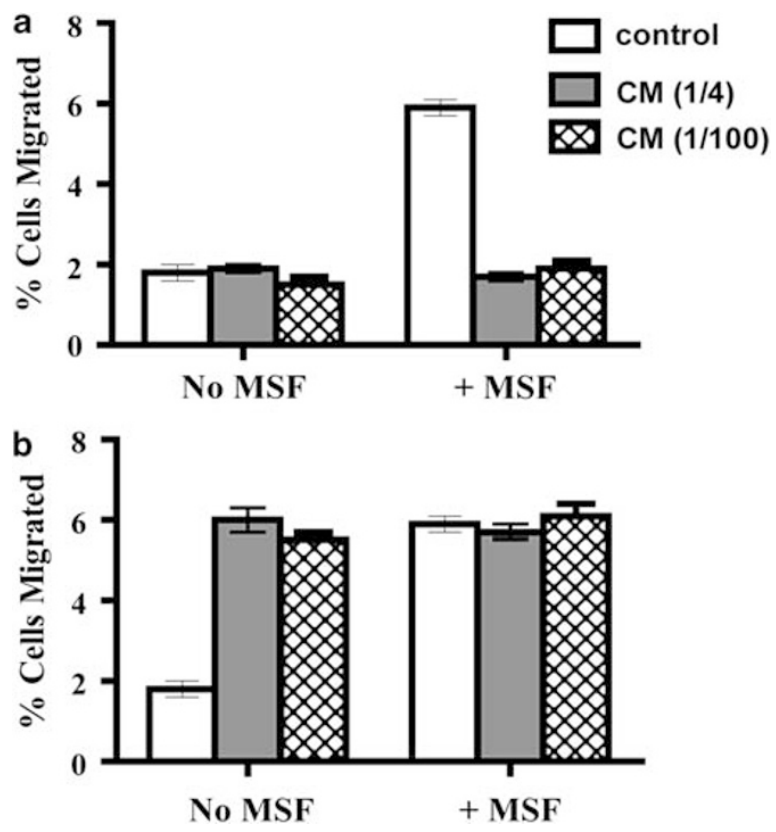

Figure 8 Endothelial cells produce a soluble inhibitor of MSF. (a) Serum-free conditioned medium (CM) was collected from a resting monolayer of an endothelial cell line derived from the human normal breast microvasculature. $\mathrm{CM}$ and control unconditioned medium (control) were tested in a fibroblast migration assay (Jones $e t \mathrm{al}^{38}$ ) in the presence or absence of MSF $(100 \mathrm{pg} / \mathrm{ml})$. The endothelial cell CM was used at a final dilution of $1 / 4$ and $1 / 100$. In control cultures, a small percentage of cells $(1.8 \pm 0.2)$ migrated into the gels in the absence of MSF. This percentage was not affected by the presence of CM. MSF stimulated cell migration to $5.9 \pm 0.2 \%$ in control medium. The presence of resting endothelial cell CM inhibited MSF-stimulated migration back to baseline levels. Purification of $\mathrm{CM}$ revealed the presence of a soluble inhibitor of MSF (MSFI) with a molecular mass initially estimated at $30 \mathrm{kDa}$. The same results were obtained using CM from other types of endothelial cells, including those derived from the bovine aorta, bovine retina, human umbilical vein, aorta, adipose tissue, and dermal microvessels. (b) CM collected from sprouting endothelial cells and tested in the same manner revealed the presence of migration-stimulating activity and absence of MSF-inhibiting activity.

including TGF- $\beta$ and NGAL, an endogenous MSF inhibitor produced by normal 'resting' keratinocytes. ${ }^{37,38}$ Interestingly, a distinct MSF inhibitor is produced by the resting endothelial cells (Figure 8a). Homogeneous monolayer cultures of such 'resting' or 'cobblestone' cells (mimicking the endothelium lining mature blood vessels in vivo) are produced by maintaining confluent endothelial cells in the absence of exogenous angiogenic factors on a 2D substratum, including the surface of a three-dimensional (3D) gel of native type I collagen fibres. ${ }^{39,40}$ A network of elongated cells displaying a 'sprouting' phenotype is rapidly induced by the addition of various angiogenic factors (such as FGF-2, MSF, and
VEGF), thereby forming a mixed culture of both resting and sprouting cells. ${ }^{39,40}$ Remarkably, endothelial cells uniformly adopt a sprouting phenotype when embedded within a 3D gel of native type I collagen in the absence of exogenous angiogenic factors (Figure 3). These

observations suggest that sprouting cells may produce an endogenous angiogenic factor(s). We confirmed that such homogeneous cultures do indeed produce bioactive MSF, but not a bioactive MSF inhibitor (Figure $8 \mathrm{~b}$ ). It thus seems that the resting endothelial cells cultured on the surface of a collagen gel may be induced to adopt a sprouting cell phenotype simply by transferring them within the 3D matrix, an example of how cell behaviour may be profoundly influenced by the physical interface between the cell and its ECM.

MSF bioactivities can also be abrogated by functionneutralising anti-MSF antibodies. ${ }^{20}$ Exposure of endothelial cells to such an antibody rapidly results in the apoptotic death of sprouting cells induced by VEGF (or other angiogenic factors), without affecting the viability of co-cultured resting cells (Figure 9). It thus seems that, in addition to inducing angiogenesis, MSF expressed by sprouting endothelial cells also acts as a convergent (downstream) autocrine survival factor for these cells.

These features of MSF biology are summarised in Figure 10 in which it is noted that (1) MSF may be expressed by several cell types, including epithelial (normal and tumour), fibroblasts, and endothelial cells, (2) MSF is a pleiotropic effector, capable of eliciting multiple responses from various target cell types, and (3) the expression of MSF and its precise effect on target cells are modulated by a hierarchy of contextual control networks involving the inter-dependent signalling of soluble factors and the ECM. In this complex framework, MSF may function in both an autocrine and a paracrine manner. The inter-dependent modulatory effects of the ECM and soluble factors on both MSF expression and target cell response are consistent with the now wellrecognised dynamic and reciprocal contextual control of cell behaviour by multiple 'tissue-level' cues. ${ }^{33,34,41,42}$ An important corollary of this understanding is that the temporal and spatial expression of MSF by its various potential producer cells, as well as the precise response (or lack of it) by its potential target cells, is not invariant, but may change in response to alterations to the tissue microenvironment during disease progression.

\section{MSF as a novel target for clinical intervention}

MSF is present in more than $80 \%$ of common human tumours. Recent data indicate that high MSF expression by tumour and tumour-associated stromal cells is associated with poor survival in patients with breast 


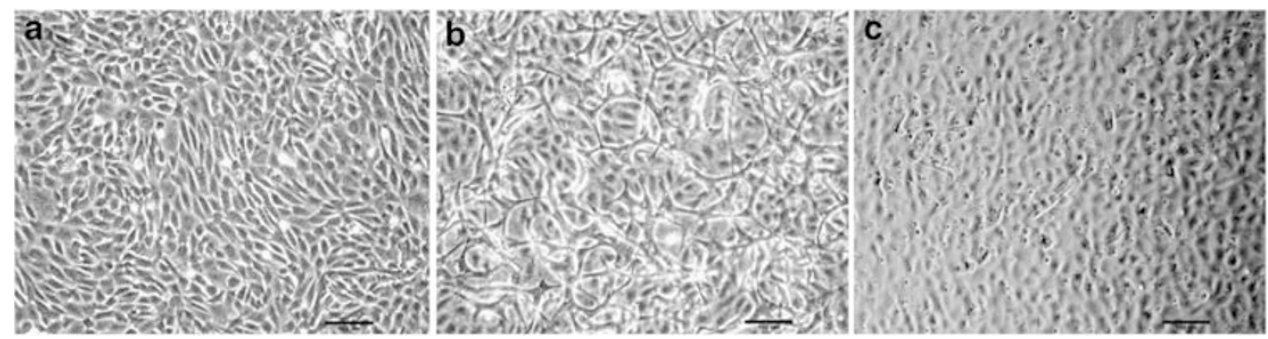

Figure 9 MSF is required for the survival of sprouting, but not resting, endothelial cells. Endothelial cells were cultured on the surface of a 3D collagen gel, as indicated in Figure 3. (a) Monolayer of resting endothelial cells in control cultures. (b) The induction of a sprouting cell network by angiogenic stimulus (such as bovine fetal serum or VEGF). (c) Selective death of sprouting cells after 1-2 days incubation with MSF function-neutralising antibody.

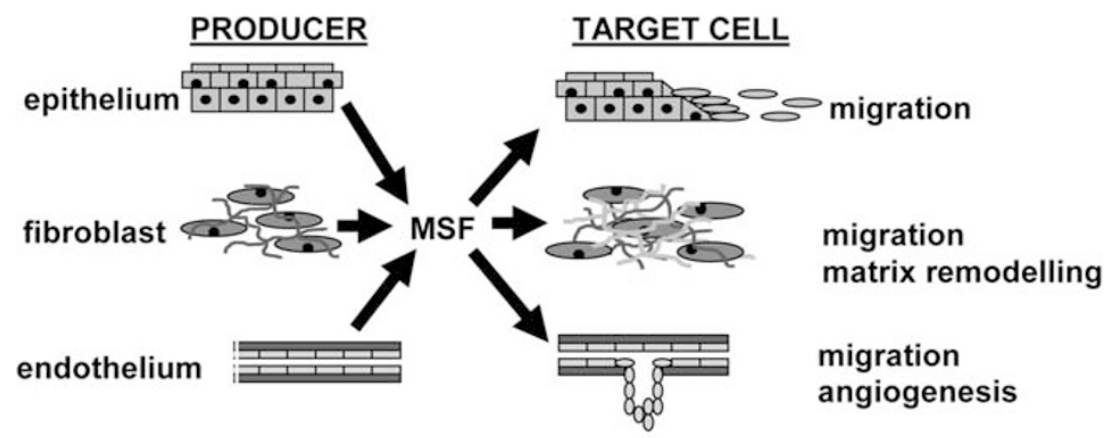

expression of and response to MSF are both

regulated by complex interplay of stimulatory and inhibitory factors (soluble and matrix)

Figure 10 Contextual control of MSF expression and effects on potential target cells. MSF may be produced by epithelial cells (normal and tumour), fibroblasts, and endothelial cells. These same cell populations are also potential targets of MSF, capable of responding in terms of the stimulation of cell migration, matrix remodelling, and angiogenesis. Control of MSF expression and manifestation of its potential bioactivities are both regulated by a complex hierarchy of inter-dependent 'contextual' actions of ECM and soluble factors.

cancer and oral cancer (unpublished observations). Such findings suggest that MSF is a novel tumour biomarker and assessment of its expression can provide independent prognostic information.

The diverse functionality of MSF is consistent with its postulated role as a driver of cancer progression. In this regard, we draw specific attention to (1) its capacity to stimulate the migration/invasion of host and tumour cells, (2) its effects on matrix remodelling, including stimulation of HA synthesis, (3) its induction of angiogenesis, and (4) its possible role as a specific survival factor for angiogenic endothelial cells. The latter finding raises the intriguing possibility of selectively inducing the destruction of tumour-induced angiogenic vessels while sparing mature host vessels. Independent evidence supporting a role for MSF in cancer pathogenesis has recently come from the study by Hu et al. ${ }^{21}$ Using an unbiased proteomic screen, these authors identified MSF as a critical angiogenic factor driving oesophageal cancer progression. Yoshino et $a l^{43}$ have additionally reported that exposure of a bronchioloalveolar carcinoma cell line to the tobacco carcinogen benzo[a]pyrene resulted in the induction of MSF expression.

Conversely, it should be noted that a failure to mount an appropriate angiogenic response contributes to the aetiology of common pathologies, such as chronic ulceration. In these situations, topical application of MSF or a synthetic agonist (such as IGDS) may help 'kick-start' the healing process. ${ }^{44}$ This possibility is supported by a pilot study, indicating that application of MSF or IGDS significantly promotes wound closure and granulation tissue neovascularisation in diabetic mice (unpublished observations).

The various facets of MSF expression and bioactivity outlined above suggest that MSF may be considered as a novel target for clinical intervention. Such intervention strategies may involve agents that modulate its reversible expression (including siRNA therapy) and/or abrogating manifestation of its bioactivities (eg, by 
function-neutralising antibodies and/or pharmacological agents). The clear influence of microenvironmental parameters in modulating both its expression and manifestation of its bioactivities offer additional potential means of clinical manipulation.

\section{Conflict of interest}

The authors declare no conflict of interest.

\section{Acknowledgements}

This study has been funded by grants from the Breast Cancer Campaign, Cancer Research UK, National Institutes of Health, USA (Grant no. 1 RO1 DK59144-01), Scottish Enterprise Proof of Concept Programme, Engineering and Physical Sciences Research Council and Biotechnology and Biological Sciences Research Council.

\section{References}

1 Folkman J, Hanahan D. Switch to the angiogenic phenotype during tumorigenesis. Princess Takamatsu Symp 1991; 22: 339-347.

2 Folkman J. The role of angiogenesis in tumor growth. Semin Cancer Biol 1992; 3: 65-71.

3 Naumov GN, Folkman J, Straume O, Akslen LA. Tumorvascular interactions and tumor dormancy. APMIS 2008; 116: 569-585.

4 Simó R, Carrasco E, García-Ramírez M, Hernández C. Angiogenic and antiangiogenic factors in proliferative diabetic retinopathy. Curr Diabetes Rev 2006; 2: 71-98.

5 Szekanecz Z, Koch AE. Mechanisms of disease: angiogenesis in inflammatory diseases. Nat Clin Pract Rheumatol 2007; 3: 635-643.

6 Friedlander M. Fibrosis and diseases of the eye. J Clin Invest 2007; 117: 576-586.

7 Ciulla TA, Rosenfeld PJ. Antivascular endothelial growth factor therapy for neovascular age-related macular degeneration. Curr Opin Ophthalmol 2009; 20: 158-165.

8 Ziemssen F, Grisanti S, Bartz-Schmidt KU, Spitzer MS. Offlabel use of bevacizumab for the treatment of age-related macular degeneration: what is the evidence? Drugs Aging 2009; 26: 295-320.

9 Carmeliet P, De Smet F, Loges S, Mazzone M. Branching morphogenesis and antiangiogenesis candidates: tip cells lead the way. Nat Rev Clin Oncol 2009; 6: 315-326.

10 Chi AS, Sorensen AG, Jain RK, Batchelor TT. Angiogenesis as a therapeutic target in malignant gliomas. Oncologist 2009; 14: 621-636.

11 Furuya M, Yonemitsu Y, Aoki III I. Angiogenesis: complexity of tumor vasculature and microenvironment. Curr Pharm Des 2009; 15: 1854-1867.

12 Economou MA. Uveal melanoma and macular degeneration: molecular biology and potential therapeutic applications. Acta Ophthalmol 2008; 86: 930-931.

13 Frank RN. Treating diabetic retinopathy by inhibiting growth factor pathways. Curr Opin Investig Drugs 2009; 10: 327-335.
14 Folkman J, Shing Y. Angiogenesis. J Biol Chem 1992; 267: 10931-10934.

15 Schor AM, Schor SL. Tumour angiogenesis: a review. J Pathol 1983; 141: 385-413.

16 Schor SL, Schor AM, Grey AM, Rushton G. Fetal and cancer patient fibroblasts produce an autocrine migrationstimulating factor not made by normal adult cells. J Cell Sci 1988; 90: 391-399.

17 Schor SL, Schor AM, Rushton G. Fibroblasts from cancer patients display a mixture of both fetal and adult-like phenotypic characteristics. J Cell Sci 1988; 90: 401-407.

18 Schor AM, Rushton G, Ferguson JE, Howell A, Redford J, Schor SL. Phenotypic heterogeneity in breast fibroblasts - functional anomaly in fibroblasts from histologically normal tissue adjacent to carcinoma. Int J Cancer 1994; 59: 25-32.

19 Schor SL, Schor AM. Stromal acceleration of tumour progression: role of fetal-like fibroblast subpopulations. Pathol Update 1997; 4: 75-95.

20 Schor SL, Ellis IR, Jones SJ, Baillie R, Seneviratne K, Clausen $\mathrm{J}$ et al. Migration-stimulating factor: a genetically truncated onco-fetal fibronectin isoform expressed by carcinoma and tumor-associated stromal cells. Cancer Res 2003; 63: 8827-8836.

$21 \mathrm{Hu}$ H, Ran Y, Zhang Y, Zhou Z, Harris SJ, Yu L et al. Antibody library-based tumor endothelial cells surface proteomic functional screen reveals migration-stimulating factor as an anti-angiogenic target. Mol Cell Proteomics 2009; 8: 816-826.

22 Hynes R. Fibronectins. Springer-Verlag: New York, 1990.

23 Kay RA, Ellis IR, Jones SJ, Perrier S, Florence MM, Schor AM et al. The expression of MSF, a potent oncofetal cytokine, is uniquely controlled by $3^{\prime}$-untranslated region dependent nuclear sequestration of its precursor messenger RNA. Cancer Res 2005; 65: 10742-10749.

24 Bakheet T, Frevel M, Williams BRG, Greer W, Khabar KSA. ARED: human AU-rich element-containing mRNA database reveals an unexpectedly diverse functional repertoire of encoded proteins. Nucleic Acids Res 2001; 29: 246-254.

25 Zhao Q, Liu X, Collodi P. Identification and characterization of a novel fibronectin in Zebrafish. Exp Cell Res 2001; 268: 211-221.

26 Liu X, Zhao Q, Collodi P. A truncated form of fibronectin is expressed in fish and mammals. Matrix Biol 2003; 22: 393-396.

27 Schor SL, Schor AM. Tumour-stroma interactions - phenotypic and genetic alterations in mammary stroma: implications for tumour progression. Breast Cancer Res 2001; 3: 373-379.

28 Schor SL, Schor AM, Grey AM, Chen J, Rushton G, Grant ME et al. Mechanism of action of the migration stimulating factor produced by fetal and cancer-patient fibroblasts: effect on hyaluronic acid synthesis. In Vitro Cell Dev Biol 1989; 25: 737-746.

29 Houard X, Germain S, Gervais M, Michaud A, van den Brule F, Foidart J-M et al. Migration-stimulating factor displays HEXXH-dependent catalytic activity important for promoting tumor cell migration. Int J Cancer 2005; 116: 378-384.

30 Schor SL, Ellis I, Banyard J, Schor AM. Motogenic activity of the IGD amino acid motif. J Cell Sci 1999; 112: 3879-3888.

31 Millard CJ, Ellis IR, Pickford AR, Schor AM, Schor SL, Campbell ID. The role of fibronectin IGD motif in 
stimulating fibroblast migration. J Biol Chem 2007; 282: 35530-35535.

32 Vakonakis I, Staunton D, Ellis IR, Starkies P, Flanagan A, Schor AM et al. Motogenic sites in human fibronectin are masked by long range interactions. J Biol Chem 2009; 284: 15668-15675.

33 Nathan C, Sporn M. Cytokines in context. J Cell Biol 1991; 113: 981-986.

34 Schor SL. Cytokine control of cell motility: modulation and mediation by the extracellular matrix. Prog Growth Factor Res 1994; 5: 223-248.

35 Pardali E, ten Dijke P. Transforming growth factor-beta signaling and tumor angiogenesis. Front Biosci 2009; 14: 4848-4861.

36 Padua D, Massague J. Role of TGF- $\beta$ in metastasis. Cell Res 2009; 19: 89-102.

37 Ellis I, Grey AM, Schor AM, Schor SL. Antagonistic effects of TGF- $\beta 1$ and MSF on fibroblast migration and hyaluronic acid synthesis - possible implications for dermal wound healing. J Cell Sci 1992; 102: 447-456.

38 Jones SJ, Florence MM, Ellis IR, Kankova K, Schor SL, Schor AM. Co-expression by keratinocytes of migration stimulating factor (MSF) and functional inhibitor of its bioactivity (MSFI). Exp Cell Res 2007; 313: 4145-4157.
39 Schor AM, Schor SL, Allen TD. The effects of culture conditions on the proliferation and morphology of bovine aortic endothelial cells in vitro: reversible expression of the sprouting cell phenotype. J Cell Sci 1983; 62: 267-285.

40 Schor AM, Ellis I, Schor SL. Collagen gel assay for angiogenesis. Induction of endothelial cell sprouting. In: Murray JC (ed). Methods in Molecular Medicine, Vol 46: Angiogenesis Protocols. Humana Press Inc: Totowa, NJ, 2001, pp 145-162.

41 Bissell MJ, Barcellos-Hoff MH. The influence of extracellular matrix on gene expression: is structure the message? J Cell Sci 1987; 8(Supp 10): 327-343.

$42 \mathrm{Xu}$ R, Boudreau A, Bissell MJ. Tissue architecture and function: dynamic reciprocity via extra- and intra-cellular matrices. Cancer Metastasis Rev 2009; 28: 167-176.

43 Yoshino I, Kometani T, Shoji F, Osoegawa A, Ohba T, Kouso $\mathrm{H}$ et al. Induction of epithelial-mesenchymal transitionrelated genes by benzo[a]pyrene in lung cancer cells. Cancer 2007; 110: 369-374.

44 Schor SL, Schor AM, Keatch RP, Belch JFF. Role of matrix macromolecules in the aetiology and treatment of chronic ulcers. In: Lee BY (ed). The Wound Management Manual. McGraw Hill: New York, Chapter 10 2005, pp 109-121. 